\title{
Patrones de reestructuración económica EN LA INDUSTRIA CHIAPANECA DE ALIMENTOS Y BEBIDAS
}

\author{
Federico Morales Barragán
}

\section{Introducción}

$\mathrm{D}$ urante los últimos años un segmento de la Industria Chiapaneca de Alimentos y Bebidas, ICHAB, ha mostrado, paulatinamente, un rostro diferente que se manifiesta con la aparición de una nueva gama de productos. Algunos de éstos son: Tortiharina, tortillas de harina de trigo condimentadas con chile jalapeño, chipilín o epazote - Tuxtla Gutiérrez y San Cristóbal de Las Casas-; Tostitadas. Tostadas de Los Altos de Chiapas — San Cristóbal de Las Casas—; los licores Tapacbulteco y Morgás — Tapachula—; Crema de café Mixcum - Tapachula—; diversos productos registrados bajo la marca Maya Gold — Tapachula_; Café MAN, café tostado y molido, también en presentación de café soluble — Tapachula_; naranjada y yogur registrados bajo la marca Spei —Comitán-; Decíspue y Arrecha, salsas - Tuxtla Gutiérrez-; Sangre de la Selva, vino generoso tipo jerez - Catazajá—; Chapé, café orgánico de sabores — Huixtla. ${ }^{1}$

Entre los rasgos más relevantes de estos productos pueden mencionarse: la diferenciación basada en su origen regional, su carácter orgánico en algunos casos o la ausencia de conservadores en otros, el cumplimiento de normas como el código de barras, la información

Federico Morales Barragán, PROIMMSE-IIA-UNAM. nutricional, las fechas de empaque y caducidad y, finalmente, su presentación mediante empaques y etiquetas cuyo diseño refleja la contratación de servicios especializados en mercadotecnia.

Este tipo de productos sugiere que un segmento de la ICHAB desarrolla un proceso de reestructuración orientado a encarar el desafío de participar en mercados, locales y foráneos, en los que actualmente se registra una competencia intensa. Por ello resulta de interés analizar la evolución económica de las actividades asociadas a estos productos.

Con base en información censal correspondiente a los años 1988, 1993 y 1998, a continuación se identifican patrones de reestructuración económica de estas actividades en cuatro municipios del estado de Chiapas: Comitán de Domínguez, San Cristóbal de Las Casas, Tapachula y Tuxtla Gutiérrez. La elección de estos municipios obedece a que en ellos se encuentra la mayoría de las empresas productoras pertenecientes a la ICHAB del tipo de bienes señalados con anterioridad. En cuanto al periodo de estudio, se determina por la periodicidad de los censos económicos. Su elección como fuente de información obedece a la posibilidad de llevar a cabo comparaciones geográficas y temporales consistentes. Es decir, la estructura de la información proporcionada por los censos económicos 
A $B$ I $E$ R $T$ T A

es homogénea para todos los municipios de la entidad y se presenta con un grado de desglose considerable, el de rama de actividad. Es de notar que la información del último censo económico, 2004, aún no se ha publicado.

El artículo se organiza como sigue: primero se identifican las actividades de la ICHAB cuyas trayectorias económicas se analizan más adelante. La denominación de las actividades se basa en la Clasificación Mexicana de Actividades y Productos, CMAP, catálogo utilizado por el Instituto Nacional de Estadística Geografía e Informática, INEGI, en los censos económicos de 1988, 1993 y 1998. ${ }^{2}$ Luego se caracterizan las trayectorias económicas de estas actividades teniendo en cuenta el análisis simultáneo de las tasas de crecimiento relativas del empleo y la productividad del trabajo. Este ejercicio permite identificar diferentes patrones de reestructuración económica de las mencionadas actividades.

\section{Ramas en reestructuración de la ICHAB}

Las actividades económicas que se analizan en este documento forman parte del subsector 31 Productos alimentarios, bebidas y tabaco, perteneciente a la Clasificación Mexicana de Actividades y Productos. La CMAP se integra por sectores, subsectores, ramas y clases, por ejemplo: Sector 3, industria manufacturera; Subsector 31, productos alimentarios, bebidas y tabaco; Rama 3112, elaboración de productos lácteos; y Clase 311203, elaboración de leche condensada, evaporada y en polvo. Incluye leche en polvo para infantes.

La tabla 1 muestra las ramas que integran el subsector que nos ocupa, 31. De sus trece ramas, en este documento se analizan siete de ellas: 3112,3113 , $3114,3115,3119,3121$ y 3130 .

Tabla 1

Ramas del subsector 31 Productos alimentarios bebidas y tabaco*

\begin{tabular}{|c|l|}
\hline Rama & \multicolumn{1}{|c|}{ Nombre } \\
\hline 3111 & Industria de la carne \\
\hline $\mathbf{3 1 1 2}$ & Elaboración de productos lácteos \\
\hline $\mathbf{3 1 1 3}$ & $\begin{array}{l}\text { Elaboración de conservas alimenticias. Incluye concentrados para caldos. Excluye las de carne } \\
\text { y leche, exclusivamente }\end{array}$ \\
\hline $\mathbf{3 1 1 4}$ & Beneficio y molienda de cereales y otros productos agrícolas \\
\hline $\mathbf{3 1 1 5}$ & Elaboración de productos de panadería \\
\hline 3116 & Molienda de nixtamal y fabricación de tortillas \\
\hline 3117 & Fabricación de aceites y grasas comestibles \\
\hline 3118 & Industria azucarera \\
\hline $\mathbf{3 1 1 9}$ & Fabricación de cocoa, chocolate y artículos de confitería \\
\hline $\mathbf{3 1 2 1}$ & Elaboración de otros productos alimentarios para el consumo humano \\
\hline 3122 & Elaboración de alimentos preparados para animales \\
\hline $\mathbf{3 1 3 0}$ & Industria de las bebidas \\
\hline 3140 & Industria del tabaco \\
\hline
\end{tabular}

*Las ramas en negritas constituyen el subconjunto que se analiza en este documento.

Fuente: INEGI (1996). 
Las ramas se seleccionaron con base en los criterios siguientes: 1) presencia de al menos un producto - vinculado a la rama de actividad - en tiendas comerciales de alguna de las ciudades en estudio: Comitán, San Cristóbal de Las Casas, Tapachula y Tuxtla; 2) los productos identificados presentan en sus empaques o etiquetas: nombre del productor establecido en Chiapas, denominación que hace explícita la diferenciación basada en el origen local del producto; código de barras; información nutricional o de la composición del producto; y fechas de caducidad y empaque.

Si bien algunos de los productos de la ICHAB son de origen orgánico y otros no contienen conservadores, este rasgo no se utilizó como criterio para seleccionar las ramas.

Para utilizar los criterios mencionados fue necesario revisar la composición de las clases de actividad que integran las ramas del subsector 31 y establecer la correspondencia entre productos y clases - véanse anexos 1 y 2 - Merece señalarse que este procedimiento presenta como limitación el hecho de que los productos tienen una correspondencia más cercana con las actividades descritas en las clases y no con las correspondientes a las ramas. De esta forma, se consideraron las ramas en las que, al menos en una de sus clases, se incluye el tipo de bienes a los que se ha hecho referencia. Por ejemplo, la rama 3114, Beneficio y molienda de cereales y otros productos agrícolas, se considera en el análisis porque incluye la clase 311403 , Tostado y molienda de café. Incluye su venta al por menor cuando esta actividad se realiza en forma conjunta con el tostado y molienda. Esta clase comprende distintas presentaciones de café, no soluble, ${ }^{3}$ comercializadas; por ejemplo bajo las marcas Maya Gold o Café MAm. En el resto de las clases que integran esta rama no se han identificado productos que cumplan los criterios señalados con anterioridad.
Si bien el análisis a nivel de ramas no tiene correspondencia uno a uno con las actividades de las empresas que elaboran el nuevo tipo de productos de la ICHAB, la caracterización de la trayectoria económica de las ramas aporta elementos significativos sobre el contexto económico específico en el que se desenvuelven dichas empresas. Además, la información a nivel de clase no está disponible a escala municipal en los censos económicos publicados.

\section{Trayectorias y patrones de reestructuración económica}

A lo largo de los últimos años distintos enfoques del análisis económico regional han insistido en la importancia de estudiar los territorios a partir de la evolución de sus transformaciones y no del estado que guardan en un momento determinado (Camagni y Capello, 1997 y 1999; Garrido, 2000; Vázquez, 1999). Esta perspectiva obedece, en buena medida, a la intensidad y variedad de las transformaciones económicas registradas, durante las últimas décadas, en diversas regiones del planeta, lo cual revela en parte su proceso de adaptación a las nuevas condiciones de la economía mundial. Estudiar la reestructuración económica de los territorios se ha convertido entonces en una tarea central del análisis regional (Llorens et al., 2002; Morgan y Nauwelaers, 2003; Nooteboom, 2000; Ratti, 1997).

Para caracterizar las trayectorias económicas de las actividades seleccionadas de la ICHAB, en este documento se utiliza un método que, de acuerdo con Garrido (2002), tiene su origen en Camagni y Cappellin (1985). Este método muestra que mediante el análisis simultáneo de las tasas de crecimiento relativas de la ocupación y de la productividad del trabajo, es posible establecer una tipología útil para caracterizar la trayectoria de las economías regionales y construir un perfil de su reestructuración económica. 
A B I E R T A

La tabla 2 muestra la matriz de trayectorias económicas que resulta de considerar simultáneamente las tasas de crecimiento relativas de la ocupación y la productividad del trabajo. El empleo de las tasas relativas permite comparar la evolución económica de las actividades municipales respecto a la de las actividades estatales. De modo que si una tasa tiene un registro mayor a la unidad, significa que la actividad municipal crece por encima del promedio representado por el registro estatal. El caso contrario también es consider

ado en la tabla mencionada.

Tabla 2

Matriz de trayectorias económicas

Tasas de crecimiento relativas de la ocupación (tcrpo) y la productividad del trabajo (tcrpr)

\begin{tabular}{|c|c|c|}
\hline tcrpo-tcrpr & $\begin{array}{c}\text { Menor que 1 } \\
(<1)\end{array}$ & $\begin{array}{c}\text { Mayor que 1 } \\
(>1)\end{array}$ \\
\hline $\begin{array}{c}\text { Menor que 1 } \\
(<1)\end{array}$ & III & II \\
\hline $\begin{array}{c}\text { Mayor que 1 } \\
(>1)\end{array}$ & Retroceso & Transformación radical \\
\hline
\end{tabular}

Fuente: Elaboración con base en Camagni y Capello, 1997, y Vázquez, 1999.

La consideración simultánea de estas tasas genera cuatro tipos de trayectorias económicas, las cuales pueden determinarse para distintos periodos. El cambio de trayectoria de una actividad municipal, por ejemplo de transformación progresiva (Iv) a expansión (I), ofrece evidencia de procesos de reestructuración económica en esa actividad. Si la trayectoria se mantiene no hay evidencia de que existan estos procesos. Lo anterior no significa que las actividades que no cambian de trayectoria no tengan modificaciones en su funcionamiento, simplemente se subraya que éstas no tienen un impacto tan importante como para alterar la trayectoria de la actividad.

La primera de las trayectorias, expansión, evidencia un desempeño favorable en términos relativos con tasas de crecimiento, en ambas variables, superiores al promedio de referencia, en este caso los registros estatales de las ramas.
Las trayectorias de transformación radical y progresiva pueden considerarse como estrategias alternativas para situarse en la trayectoria de expansión. La primera presenta un crecimiento de la productividad que afecta en forma drástica los niveles de ocupación, pero se espera que propicie una evolución favorable del producto. La transformación progresiva, por su parte, refleja un proceso de ajuste paulatino hacia la expansión que privilegia el impulso al empleo, pero también puede indicar la presencia de mecanismos de subvención que estimulan el crecimiento del empleo sin impulsar la expansión de la productividad del trabajo.

Finalmente, en la trayectoria de retroceso la disminución de la ocupación no afecta positivamente a la productividad del trabajo y con ello a la competitividad, lo que establece una situación en la que empeoran tanto la ocupación como el producto (Camagni y Capello, 1997 y 1999; Vázquez, 1999). 
A continuación se presentan las trayectorias y patrones de reestructuración económica de cada una de las siete ramas seleccionadas en los municipios de Comitán, San Cristóbal, Tapachula y Tuxtla Gutiérrez, para los periodos 1988-1993 y 1993-1998. ${ }^{4}$

\section{Elaboración de productos lácteos (3112)}

La información del cuadro 1 revela la heterogeneidad de los procesos de reestructuración económica de la rama. La caracterización de las trayectorias permite identificar tres patrones diferentes de reestructuración. Comitán y San Cristóbal presentan el cambio más significativo, pues transitan de una trayectoria de retroceso (III) a una de expansión (I). Es decir, en ambas municipios esta rama registra, en el segundo periodo, crecimientos de la ocupación y la productividad del trabajo superiores a los del estado de Chiapas. En relación con Tapachula y Tuxtla Gutiérrez, la reestructuración parte de una situación similar en la que se privilegia el crecimiento de la ocupación y no de la productividad (IV), pero la evolución es diferente. Tapachula llega a una situación caracterizada por el impulso de la productividad del trabajo (II), mientras que Tuxtla cae en una trayectoria de retroceso (III). Por ello, como lo señala el cuadro 1, el patrón de reestructuración de Tuxtla se considera fracaso de una estrategia que privilegia el empleo sobre la productividad (IV).

\section{Cuadro 1}

Trayectorias y patrones de reestructuración de la rama 3112

\begin{tabular}{|c|c|c|c|c|}
\hline Municipio & Región & $\begin{array}{c}\text { Trayectoria } \\
1988-1993\end{array}$ & $\begin{array}{c}\text { Trayectoria } \\
1993-1998 \\
\end{array}$ & Patrón de reestructuración \\
\hline Comitán & Fronteriza & III & $\mathrm{I}$ & \multirow{2}{*}{$\begin{array}{c}\text { Reestructuración drástica hacia } \\
\text { la expansión }\end{array}$} \\
\hline San Cristóbal de Las Casas & Altos & III & $\mathrm{I}$ & \\
\hline Tapachula & Soconusco & IV & II & $\begin{array}{c}\text { De la transformación progresiva } \\
\text { a la radical }\end{array}$ \\
\hline Tuxtla Gutiérrez & Centro & IV & III & $\begin{array}{c}\text { Fracaso de la transformación } \\
\text { progresiva }\end{array}$ \\
\hline
\end{tabular}

Fuente: Elaboración propia con base en los resultados del sistema Indica 1.0

Elaboración de conservas alimenticias. Incluye concentrados para caldos. Excluye las de carne y leche, exclusivamente (3113)

Como se mencionó en la segunda sección de este artículo, cuando una actividad mantiene su trayectoria, como es el caso de esta rama, se considera que no hay indicios de procesos de reestructuración. Lo anterior no significa la inexistencia de cambios, simplemente que su intensidad no modifica la trayectoria de la actividad. Como se aprecia en el cuadro 2, la rama
3113 crece a un menor ritmo que el promedio estatal, tanto en lo que refiere a la ocupación como a la productividad del trabajo. Además esta actividad aparece sólo en el municipio de Tapachula. Es decir, en este municipio se dispone de registros para los tres años, mientras que en Comitán hay sólo información para el año 1998. En San Cristóbal y Tuxtla no hay registro censal de esta actividad. 
Cuadro 2

Trayectorias y patrones de reestructuración de la rama 3113

\begin{tabular}{|c|c|c|c|c|}
\hline Municipio & Región & $\begin{array}{c}\text { Trayectoria } \\
1988-1993\end{array}$ & $\begin{array}{c}\text { Trayectoria } \\
1993-1998\end{array}$ & Patrón de reestructuración \\
\hline Tapachula & Soconusco & III & III & \\
\hline
\end{tabular}

Fuente: Elaboración propia con base en los resultados del sistema Indica 1.0.

\section{Cuadro 3}

Trayectorias y patrones de reestructuración de la rama 3114

\begin{tabular}{|c|c|c|c|c|}
\hline Municipio & Región & $\begin{array}{c}\text { Trayectoria } \\
\mathbf{1 9 8 8 - 1 9 9 3}\end{array}$ & $\begin{array}{c}\text { Trayectoria } \\
\text { 1993-1998 }\end{array}$ & Patrón de reestructuración \\
\hline Comitán & Fronteriza & IV & II & $\begin{array}{c}\text { De la transformación progresiva } \\
\text { a la radical }\end{array}$ \\
\hline San Cristóbal de Las Casas & Altos & IV & III & $\begin{array}{c}\text { Fracaso de la transformación } \\
\text { progresiva }\end{array}$ \\
\hline Tapachula & Soconusco & I & III & $\begin{array}{c}\text { Reestructuración drástica hacia el } \\
\text { retroceso }\end{array}$ \\
\hline Tuxtla Gutiérrez & Centro & III & II & $\begin{array}{c}\text { Del retroceso a la transformación } \\
\text { radical }\end{array}$ \\
\hline
\end{tabular}

Fuente: Elaboración propia con base en los resultados del sistema Indica 1.0.

\section{Cuadro 4}

Trayectorias y patrones de reestructuración de la rama 3115

\begin{tabular}{|c|c|c|c|c|}
\hline Municipio & Región & $\begin{array}{c}\text { Trayectoria } \\
\text { 1988-1993 }\end{array}$ & $\begin{array}{c}\text { Trayectoria } \\
\mathbf{1 9 9 3 - 1 9 9 8}\end{array}$ & Patrón de reestructuración \\
\hline Comitán & Fronteriza & II & IV & $\begin{array}{c}\text { De la transformación radical a la } \\
\text { progresiva }\end{array}$ \\
\hline San Cristóbal de Las Casas & Altos & I & IV & $\begin{array}{c}\text { De la expansión a la } \\
\text { transformación progresiva }\end{array}$ \\
\hline Tapachula & Soconusco & II & I & $\begin{array}{c}\text { Exito de la transformación } \\
\text { radical }\end{array}$ \\
\hline Tuxtla Gutiérrez & Centro & II & IV & $\begin{array}{c}\text { De la transformación radical a la } \\
\text { progresiva }\end{array}$ \\
\hline
\end{tabular}

Fuente: Elaboración propia con base en los resultados del sistema Indica 1.0. 
Beneficio y molienda de cereales y otros productos agrícolas (3114)

La heterogeneidad de los patrones de reestructuración es un rasgo que también caracteriza la evolución de esta rama: cuatro patrones diferentes en cuatro municipios. El funcionamiento de la rama revela, además, la utilidad de caracterizar la evolución de las actividades y no sólo su situación en un momento determinado. Lo anterior muestra el valor de la noción de patrón de reestructuración. En el cuadro 3 se aprecia que en Tapachula y San Cristóbal se arriba a una trayectoria de retroceso (III), sin embargo, los patrones de reestructuración respectivos son diferentes. En el primer caso el municipio parte de una trayectoria de expansión (I), mientras que en el segundo la trayectoria de partida muestra un mayor dinamismo del empleo (IV). Una reflexión similar se deriva de considerar los diferentes patrones de Comitán y Tuxtla Gutiérrez. En ambos municipios se registra, en el segundo periodo, un mayor dinamismo de la productividad del trabajo (II), pero es de notar la diferencia significativa de sus trayectorias de partida (IV) y (III), respectivamente. Es decir, el patrón de Comitán revela el cambio de privilegiar el empleo por la productividad del trabajo, mientras que el de Tuxtla muestra un mayor dinamismo de la productividad del trabajo.

\section{Elaboración de productos}

de panadería (3115)

En el cuadro 4 se aprecian tres patrones de reestructuración económica. Los municipios de Comitán y 'Tuxtla registran una evolución similar; en ambos casos se transita de un mayor dinamismo de la productividad del trabajo hacia un mayor dinamismo de la ocupación (II-IV). El patrón de reestructuración de San Cristóbal muestra una pérdida de dinamismo en la productividad del trabajo, pero conserva la tendencia favorable de crecimiento del empleo (I-IV). Por último, el patrón de reestructuración de Tapachula revela con nitidez el éxito de la estrategia de impulso a la productividad del trabajo (II), pues en el segundo periodo se arriba a una trayectoria de expansión (I).

Fabricación de cocoa, chocolate

y artículos de confitería (3119)

De los tres municipios que aparecen en el cuadro 5, sólo en Tuxtla Gutiérrez puede identificarse un patrón de reestructuración, lo cual significa que hay registros de la actividad en los años 1988, 1993 y 1998. En relación con el patrón de este municipio se aprecia con claridad que al escaso dinamismo relativo del empleo (II), en el primer periodo, se une, entre 1993 y 1998, un comportamiento similar en la productividad del trabajo, de modo que la rama se ubica en la trayectoria de retroceso (III). En Comitán y San Cristóbal no hay registros de esta rama para 1988, lo cual impide establecer sus trayectorias para el primer periodo. Atendiendo al primer caso, la trayectoria muestra el mayor dinamismo de la productividad del trabajo respecto a la ocupación (II), mientras que en San Cristóbal se registra un comportamiento de la productividad del trabajo y el empleo inferior al del promedio estatal, de ahí su ubicación en la trayectoria de retroceso (III). Merece señalarse que para el municipio de Tapachula únicamente se registra información censal en 1993, de modo que no es posible establecer sus trayectorias económicas.

\section{Elaboración de otros productos alimentarios} para el consumo humano (3121)

La rama 3121 muestra el mismo rasgo de heterogeneidad que se ha observado en las ramas anteriores. El patrón más favorable se registra en Comitán, donde se mantiene, a lo largo del periodo analizado, una trayectoria de expansión (I), esto es, crecimiento de la productividad del trabajo y la ocupación con ritmos superiores a los de Chiapas. En Tuxtla Gutiérrez la trayectoria final también se expande 
A $B$ I $E$ R $T$ T

\section{Cuadro 5}

Trayectorias y patrones de reestructuración de la rama 3119

\begin{tabular}{|c|c|c|c|c|}
\hline Municipio & Región & $\begin{array}{c}\text { Trayectoria } \\
\mathbf{1 9 8 8 - 1 9 9 3}\end{array}$ & $\begin{array}{c}\text { Trayectoria } \\
\mathbf{1 9 9 3 - 1 9 9 8}\end{array}$ & Patrón de reestructuración \\
\hline Comitán & Fronteriza & & II & \multirow{2}{*}{} \\
\cline { 1 - 4 } San Cristóbal de Las Casas & Altos & & III & \multirow{2}{*}{ Fracaso de la transformación } \\
radical
\end{tabular}

Fuente: Elaboración propia con base en los resultados del sistema Indica 1.0.

\section{Cuadro 6}

Trayectorias y patrones de reestructuración de la rama 3121

\begin{tabular}{|c|c|c|c|c|}
\hline Municipio & Región & $\begin{array}{c}\text { Trayectoria } \\
\text { 1988-1993 }\end{array}$ & $\begin{array}{c}\text { Trayectoria } \\
\mathbf{1 9 9 3 - 1 9 9 8}\end{array}$ & Patrón de reestructuración \\
\hline Comitán & Fronteriza & I & I & Mantienen su trayectoria \\
\hline San Cristóbal de Las Casas & Altos & I & IV & $\begin{array}{c}\text { De la expansión a la } \\
\text { transformación progresiva }\end{array}$ \\
\hline Tapachula & Soconusco & IV & II & $\begin{array}{c}\text { De la transformación progresiva } \\
\text { a la radical }\end{array}$ \\
\hline Tuxtla Gutiérrez & Centro & IV & I & $\begin{array}{c}\text { Éxito de la transformación } \\
\text { progresiva }\end{array}$ \\
\hline
\end{tabular}

Fuente: Elaboración propia con base en los resultados del sistema Indica 1.0.

\section{Cuadro 7}

Trayectorias y patrones de reestructuración de la rama 3130

\begin{tabular}{|c|c|c|c|c|}
\hline Municipio & Región & $\begin{array}{c}\text { Trayectoria } \\
\mathbf{1 9 8 8 - 1 9 9 3}\end{array}$ & $\begin{array}{c}\text { Trayectoria } \\
\mathbf{1 9 9 3 - 1 9 9 8}\end{array}$ & Patrón de reestructuración \\
\hline Comitán & Fronteriza & IV & II & $\begin{array}{c}\text { De la transformación progresiva } \\
\text { a la radical }\end{array}$ \\
\hline San Cristóbal de Las Casas & Altos & I & III & $\begin{array}{c}\text { Reestructuración drástica hacia el } \\
\text { retroceso }\end{array}$ \\
\hline Tapachula & Soconusco & II & IV & $\begin{array}{c}\text { De la transformación radical a la } \\
\text { progresiva }\end{array}$ \\
\hline Tuxtla Gutiérrez & Centro & III & I & $\begin{array}{c}\text { Reestructuración drástica hacia la } \\
\text { expansión }\end{array}$ \\
\hline
\end{tabular}

Fuente: Elaboración propia con base en los resultados del sistema Indica 1.0. 
(I) y se constata el éxito de la transformación progresiva (Iv), trayectoria registrada en el periodo inicial. En Tapachula se aprecia un cambio en la variable que muestra mayor dinamismo. En el primer periodo, el dinamismo se registra en la ocupación (IV), mientras que en el segundo se traslada a la productividad del trabajo (II). Por último, el patrón de reestructuración de San Cristóbal muestra una pérdida de dinamismo de la productividad del trabajo, pues de una trayectoria de expansión (I) pasa a una de transformación progresiva (IV), en la cual la productividad del trabajo crece a un ritmo inferior al del promedio estatal. Cuadro 6.

\section{Industria de las bebidas (3130)}

En esta rama se aprecia un comportamiento peculiar, pues se observa que en dos municipios ocurre una reestructuración drástica, aunque de signo diferente: San Cristóbal y Tuxtla. Mientras que en los otros dos municipios, Comitán y Tapachula, se aprecian cambios opuestos en sus patrones de reestructuración. En San Cristóbal hay una pérdida de dinamismo tanto en la productividad del trabajo como en la ocupación, así lo muestra el paso desde la expansión (I) al retroceso (III). El comportamiento contrario se registra en Tuxtla (III-I), donde la ocupación y la productividad del trabajo crecen, en el segundo periodo, a un ritmo superior a los promedios estatales. En Comitán y Tapachula los patrones de reestructuración respectivos también tienen signo contrario. Es decir, en el primer caso la evolución se da a favor de la productividad del trabajo (IV-II), mientras que en Tapachula la evolución se da en sentido inverso, esto es, a favor de la ocupación (II-IV). Cuadro 7.

\section{Reflexiones finales}

La identificada diversidad de patrones de reestructuración en este artículo muestra la heterogeneidad en la evolución económica de las ramas incluidas en la ICHAB. El análisis desarrollado revela la presencia de diez patrones de reestructuración de los doce posibles que se derivan del análisis de trayectorias económicas propuesto — véase anexo 3, tablas 3 y 4.

Destaca también que ninguno de los diez patrones identificados predomina en la evolución económica de las ramas. De los veinticuatro casos analizados, el patrón que registra mayor frecuencia, con cuatro casos, es el que transita de la transformación progresiva a la radical (IV-II). Es decir, la evolución económica de las ramas en esta situación se desplaza del dinamismo en el empleo al dinamismo en la productividad del trabajo. Esta circunstancia se presenta en los municipios de Comitán y Tapachula: para el primero en las ramas 3114 y 3130, para el segundo en las ramas 3112 y 3121.

Lo anterior muestra además la inexistencia de una tendencia para alguna de las ramas. Una misma rama presenta en general patrones de reestructuración diferentes en cada municipio. Las excepciones a esta condición se registran en las ramas 3112 y 3115 . La primera presenta el patrón de reestructuración drástica hacia la expansión (III-I) en los municipios de Comitán y San Cristóbal. Mientras que la rama 3115 presenta el patrón que se desplaza de la transformación radical a la progresiva (II-IV) en Comitán y Tuxtla Gutiérrez. Cada una de las cinco ramas restantes - 3113, 3114, 3119, 3121, 3130 - manifiesta una evolución económica diferente en cada uno de los municipios en los que se encuentra presente.

Un comentario particular toma en cuenta los casos en los que no se identifican patrones de reestructuración económica. Un primer caso se encuentra en las ramas que no modifican su trayectoria; como se ha insistido anteriormente, esta situación no significa ausencia de cambios, sino que éstos no tienen la intensidad suficiente para modificar las trayectorias iniciales de las ramas. En esta situación se encuentran las ramas 3121 en Comitán, con una trayectoria de expansión en ambos periodos (I-I), y la 3113 en Tapachula, con una trayectoria de retroceso en ambos periodos (III-III). Un 
segundo caso refiere a las ramas para las cuales no existen registros en alguno de los tres años que se estudian. Estas ramas son la 3113 y 3119 . En la primera, Comitán sólo muestra registro para 1998, San Cristóbal y Tuxtla Gutiérrez carecen de registros en los tres años considerados. Respecto a la rama 3119, Comitán y San Cristóbal cuentan con registros en 1993 y 1998, y en Tapachula se dispone de información sólo para 1993.

Los resultados anteriores señalan la pertinencia de valorar las trayectorias de las actividades económicas y no sólo el estado que guardan en un momento determinado. Una actividad puede registrar una situación de expansión, pero la valoración de este hecho varía si su estado previo también es de expansión o de retroceso, o bien, si la expansión es fruto de una estrategia que privilegia el impulso al empleo y no la productividad del trabajo, o al revés.

La caracterización de las trayectorias económicas de estas actividades permite además identificar los patrones de reestructuración que las mismas despliegan. La información suministrada en este artículo revela que no existen rutas predeterminadas en la estructuración de estos patrones. Por ejemplo, un mayor dinamismo relativo de la productividad del trabajo no conduce necesariamente a una trayectoria de expansión. Lo anterior muestra la presencia de factores específicos, que de cierta forma violentan "hechos estilizados" como el anterior, referidos a los ámbitos macro, meso y micro-económicos que merecen ser considerados para explicar la evolución de estas actividades productivas. A ello puede contribuir, por ejemplo, el uso de técnicas como la denominada shift \& share — de cambio y participación-, mediante la cual puede valorarse la evolución de la especialización económica estatal y municipal en la configuración de los patrones de reestructuración identificados. O bien, la utilización de metodologías orientadas a explorar la interrelación entre el desempeño de las unidades económicas y el ambiente institucional en el que se ubican (MeyerStamer, 1998, 2000).

\section{Anexo 1 \\ Subsector 31 Productos alimentarios, bebidas y tabaco \\ Ramas seleccionadas \\ 3112 Elaboración de productos lácteos}

Clase 311201 Tratamiento y envasado de leche. Comprende pasteurización, homogeneización, etcétera. Incluye distribución cuando la realizan los establecimientos productores.

Clase 311202 Elaboración de crema, mantequilla y queso. Incluye producción de caseína, lactosa y suero. Incluye venta al por menor cuando la actividad se realiza en forma conjunta con la elaboración de estos productos.

Clase 311203 Elaboración de leche condensada, evaporada y en polvo. Incluye leche en polvo para infantes.

Clase 311204 Elaboración de helados y paletas. Incluye los de leche y agua. Excluye a los establecimientos que sólo se dedican a la comercialización, y que se clasifican en la rama 6140 o 6210, según sea el caso.

Clase 311205 Elaboración de cajetas y otros productos lácteos. Incluye yogures y alimentos a base de leche para niños y enfermos; flanes y postres a base de leche, ya preparados.

\section{Elaboración de conservas alimenticias. Incluye concentrados para caldos. Excluye las de carne y leche, exclusivamente}

Clase 311301 Preparación y envasado de frutas y legumbres. Incluye congelación y encurtido, jugos y mermeladas de frutas, así como conservas y alimentos preparados para enfermos y niños que no tengan leche. 
Excluye los servicios agrícolas de empaque y envasado que se clasifican en la clase 971010, "prestación de servicios agrícolas".

Clase 311302 Deshidratación de frutas y legumbres. Clase 311303 Elaboración de sopas y guisos preparados. Incluye salsas con tomate.

Clase 311304 Congelación y empaque de pescados y mariscos frescos.

Clase 311305 Preparación y envasado de conservas de pescados y mariscos. Incluye algas marinas enlatadas, mariscos ahumados enlatados, huevos y residuos de pescado y pieles sin curtir de peces y mamíferos marinos.

Clase 311306 Secado y salado de pescados y mariscos. Incluye pescado y mariscos ahumados y camarón en polvo.

Clase 311307 Elaboración de concentrados para caldos de carne de res, pollo, pescado, mariscos y verduras.

\section{Beneficio y molienda de cereales y otros productos agrícolas}

Clase 311401 Beneficio de arroz. Comprende descascarado, limpieza, pulido, y envasado cuando se realizan como actividades complementarias al beneficio. Clase 311402 Beneficio de café.

Clase 311403 Tostado y molienda de café. Incluye su venta al por menor cuando esta actividad se realiza en forma conjunta con el tostado y molienda.

Clase 311404 Molienda de trigo. Incluye la elaboración de harina de trigo.

Clase 311405 Elaboración de harina de maíz. Incluye la elaboración industrial de harina para tamales. Incluye la elaboración de fécula de maíz cuando ésta sea elaborada por el establecimiento productor de la harina. Excluye los molinos de nixtamal que se encuentran clasificados en la clase 311601, "molienda de nixtamal".
Clase 311406 Elaboración de otros productos de molino que contengan cereales y leguminosas. Incluye harinas. Incluye la elaboración de harina de frijol, soya, arroz, algodón, avena molida, cebada molida, hojuela molida, etcétera. Excluye de trigo o de maíz que se clasifican en las clases 311404, "molienda de trigo", y 311405, "elaboración de harina de maíz".

Clase 311407 Beneficio de otros productos agrícolas no mencionados anteriormente. Incluye limpieza y envasado de frijol, habas, lentejas, etc., cuando se realizan como actividades complementarias al beneficio. Excluye el beneficio de cacao que se clasifica en la clase 311901, "elaboración de cocoa y chocolate de mesa".

\section{Elaboración de productos de panadería}

Clase 311501 Elaboración de galletas y pastas alimenticias. Incluye la elaboración de tortillas de harina. Clase 311502 Elaboración y venta de pan y pasteles - panaderías-. Incluye canapés. Excluye a los expendios revendedores que se clasifican en la clase 621011, "comercio al por menor de pan y tortillas". Clase 311503 Panadería y pastelería industrial. Incluye los productos elaborados en plantas industriales con alta utilización de maquinaria.

\section{Fabricación de cocoa, chocolate y artículos de confitería}

Clase 311901 Elaboración de cocoa y chocolate de mesa. Incluye beneficio de cacao.

Clase 311902 Elaboración de dulces, bombones y confituras. Incluye ates, frutas cubiertas y otros dulces regionales.

Clase 311903 Fabricación de chicles. 


\section{Elaboración de otros productos alimentarios para el consumo humano}

Clase 312110 Elaboración de café soluble. Incluye extractos y concentrados de café.

Clase 312121 Elaboración de concentrados, jarabes y colorantes naturales para alimentos. Incluye la elaboración de refrescos en polvo. Excluye la fabricación de colorantes sintéticos para alimentos que se clasifican en la clase 351213, "fabricación de colorantes y pigmentos".

Clase 312122 Tratamiento y envasado de miel de abeja. Clase 312123 Elaboración de almidones, féculas y levaduras. Incluye gluten, azúcar de uva y de maíz. Clase 312124 Elaboración de mayonesa, vinagre y otros condimentos. Incluye refinación de sal. También mole, mostaza, salsas de chile — molienda de chile-, salsas a base de mayonesas, sazonadores, especias molidas, etcétera.

Clase 312125 Fabricación de hielo. Excluye hielo seco o artificial que se clasifica en la clase 351216, "fabricación de otros productos químicos básicos".

Clase 312126 Elaboración de gelatinas, flanes y postres en polvo para preparar en el hogar. Incluye la elaboración de grenetina comestible. Excluye los flanes y postres que contengan leche, ya preparados, que se clasifican en la clase 311205, "elaboración de cajetas y otros productos lácteos"; y las gelatinas de agua ya preparadas, en la clase 312129, "elaboración de otros productos alimenticios para consumo humano".

Clase 312127 Elaboración de botanas y productos de maíz no mencionados anteriormente. Incluye papas fritas, charritos, chicharrones, palomitas, frituras de maíz, etcétera.
Clase 312128 Envasado de té. Incluye extractos y concentrados de té.

Clase 312129 Elaboración de otros productos alimentarios para consumo humano. Incluye la elaboración de gelatinas de agua ya preparadas y azúcar glass.

\section{Industria de las bebidas}

Clase 313011 Elaboración de bebidas destiladas de agaves.

Clase 313012 Elaboración de bebidas destiladas de caña.

Clase 313013 Elaboración de bebidas destiladas de uva. Incluye brandy, coñac y otros.

Clase 313014 Elaboración de otras bebidas alcohólicas destiladas. Incluye vodka, ginebra, whisky, rompope y licores.

Clase 313020 Destilación de alcohol etílico. Incluye el proceso de desnaturalización del alcohol. Cuando su producción se encuentra completamente integrada a la producción de azúcar y de caña se clasifica en la clase 311801, "elaboración de azúcar y productos residuales de la caña".

Clase 313031 Vinificación —elaboración de bebidas fermentadas de uva —. Incluye vinos de mesa, vermú y otros.

Clase 313032 Elaboración de pulque.

Clase 313033 Elaboración de sidra.

Clase 313040 Industria de la cerveza y la malta.

Clase 313050 Elaboración de refrescos y otras bebidas no alcohólicas. Incluye la elaboración de aguas minerales y purificadas. 


\section{Ramas no seleccionadas}

\section{Industria de la carne}

Clase 311101 Matanza de ganado y aves. Comprende rastros y obradores, incluyendo sus desechos y extractos. Clase 311102 Congelación y empacado de carne fresca. Clase 311104 Preparación de conservas y embutidos de carne. Incluye subproductos como fundición de sebo. Excluye a los establecimientos que sólo se dedican a la comercialización y que se clasifican en la rama 6140 o 6210, según sea el caso.

\section{Molienda de nixtamal y fabricación de tortillas}

Clase 311601 Molienda de nixtamal. Se incluyen los establecimientos que se dedican exclusivamente a la molienda — molinos de nixtamal.

Clase 311602 Tortillerías. Excluye a los expendios revendedores que se clasifican en la clase 621011, "comercio al por menor de pan y tortillas".

\section{Fabricación de aceites y grasas comestibles}

Clase 311701 Fabricación de aceites y grasas vegetales comestibles. Incluye pastas y harina de semillas oleaginosas y subproductos. Excluye la elaboración de aceites esenciales que se clasifican en la clase 352238, "fabricación de aceites esenciales".

Clase 311702 Fabricación de grasas y aceites animales comestibles. Incluye la elaboración de la manteca de cerdo y res cuando sean el producto principal. Excluye la elaboración de grasas y aceites para usos medicinales que se clasifican en la rama 3521, "industria farmacéutica”; así como la refinación de grasas y aceites animales no comestibles que se clasifican en la clase 352239, "refinación de grasas y aceites animales no comestibles".

\section{Industria azucarera}

Clase 311801 Elaboración de azúcar y productos residuales de la caña. Incluye la destilación de alcohol etílico cuando esta actividad se encuentra completamente integrada a las labores de los establecimientos productores de azúcar de caña. Clase 311802 Elaboración de piloncillo o panela.

\section{Elaboración de alimentos preparados para animales}

Clase 312200 Preparación y mezcla de alimentos para animales. Incluye forrajes preparados. Incluso harinas de pescado, de cempasúchitl, de sangre y plumas, etcétera.

\section{Industria del tabaco}

Clase 314001 Beneficio de tabaco. Incluye las labores de desecación y clasificación después de efectuada la subasta del mismo. Excluye las labores previas a la subasta que se clasifican en la clase 971010, "prestación de servicios agrícolas".

Clase 314002 Fabricación de cigarros.

Clase 314003 Fabricación de puros y otros productos de tabaco. Incluye rapé, tabaco para mascar y para pipa. 
Anexo 2

Muestra de los nuevos productos de la ICHAB

\begin{tabular}{|c|c|c|c|}
\hline Producto/productor & $\begin{array}{c}\text { Lugar de } \\
\text { producción }\end{array}$ & Rama & Clase \\
\hline $\begin{array}{c}\text { Leche Pradel/Lácteos de Chiapas, SA } \\
\text { de CV }\end{array}$ & Berriozábal & 3112 & $\begin{array}{l}311201 \text { Tratamiento y envasado de leche. } \\
\text { Comprende su pasteurización, homoge- } \\
\text { neización, etcétera. Incluye su distribución } \\
\text { cuando la realizan los establecimientos } \\
\text { productores. }\end{array}$ \\
\hline $\begin{array}{l}\text { Yoghurt Spei/Pérez Castellanos y } \\
\text { Compañía S de R L M I }\end{array}$ & Comitán & 3112 & $\begin{array}{l}311205 \text { Elaboración de cajetas y otros } \\
\text { productos lácteos. Incluye yogures y alimentos } \\
\text { con base de leche para niños y enfermos; } \\
\text { flanes y postres a base de leche, ya preparados. }\end{array}$ \\
\hline $\begin{array}{c}\text { Mango deshidratado Maya } \\
\text { Gold/Productos Naturales de la } \\
\text { Región Maya de Chiapas, SA de CV }\end{array}$ & Tapachula & 3113 & 311302 Deshidratación de frutas y legumbres. \\
\hline $\begin{array}{c}\text { Café Mam orgánico } \\
\text { (Gourmet)/Indígenas de la Sierra } \\
\text { Madre de Motozintla "San Isidro } \\
\text { Labrador", Sociedad de Solidaridad } \\
\text { Social } \\
\text { Café gourmet Maya Gold/Productos } \\
\text { Naturales de la Región Maya de } \\
\text { Chiapas, SA de CV }\end{array}$ & Tapachula & 3114 & $\begin{array}{l}311403 \text { Tostado y molienda de café. Incluye su } \\
\text { venta al por menor cuando esta actividad se } \\
\text { realiza en forma conjunta con el tostado y } \\
\text { molienda. }\end{array}$ \\
\hline $\begin{array}{l}\text { Tortiharina, tortillas de harina de trigo } \\
\text { condimentadas con chile jalapeño, } \\
\text { chipilín o epazote }\end{array}$ & $\begin{array}{c}\text { San Cristóbal } \\
\text { de Las Casas, } \\
\text { Tuxtla } \\
\text { Gutiérrez }\end{array}$ & 3115 & $\begin{array}{l}311501 \text { Elaboración de galletas y pastas } \\
\text { alimenticias. Incluye la elaboración de tortillas } \\
\text { de harina. }\end{array}$ \\
\hline & & 3119 & \\
\hline $\begin{array}{c}\text { Crema de café Mixcum/Agro Izapa S } \\
\text { de RL MI }\end{array}$ & Tapachula & 3121 & $\begin{array}{l}312110 \text { Elaboración de café soluble. Incluye } \\
\text { extractos y concentrados de café. }\end{array}$ \\
\hline $\begin{array}{l}\text { Salsa Arrecha/Cancino Huerta } \\
\text { Alimentos Procesados }\end{array}$ & $\begin{array}{l}\text { Tuxtla } \\
\text { Gutiérrez }\end{array}$ & 3121 & $\begin{array}{l}312124 \text { Elaboración de mayonesa, vinagre y } \\
\text { otros condimentos. Incluye refinación de sal. } \\
\text { Incluye mole, mostaza, salsas de chile } \\
\text { (molienda de chile), salsas de mayonesas, } \\
\text { sazonadores, especias molidas, etcétera. }\end{array}$ \\
\hline $\begin{array}{c}\text { Tostadin/Juan Manuel Ruiz Trinidad } \\
\text { Tostitadas/Tostadas de Los Altos de } \\
\text { Chiapas } \\
\text { Chicharrón de puerco Rincón } \\
\text { Tarasco/Frituras Rincón Tarasco SA } \\
\text { de CV }\end{array}$ & $\begin{array}{l}\text { San Cristóbal } \\
\text { de Las Casas }\end{array}$ & 3121 & $\begin{array}{l}312127 \text { Elaboración de botanas y productos de } \\
\text { maíz no mencionados anteriormente. Incluye } \\
\text { papas fritas, charritos, chicharrones, palomitas, } \\
\text { frituras de maíz, etcétera. }\end{array}$ \\
\hline $\begin{array}{c}\text { Naranjada Spei/ Pérez Castellanos y } \\
\text { Compañía S de R L M I }\end{array}$ & Comitán & 3130 & $\begin{array}{l}\text { Clase } 313050 \text { Elaboración de refrescos y otras } \\
\text { bebidas no alcohólicas. Incluye la elaboración } \\
\text { de aguas minerales y purificadas. }\end{array}$ \\
\hline
\end{tabular}

Fuente: Elaboración propia con base en investigación inicial de mercado. 


\section{Anexo 3}

Tabla 3

Trayectorias y patrones de reestructuración económica

\begin{tabular}{|c|c|c|}
\hline $\begin{array}{c}\text { Trayectoria } \\
1988-1993\end{array}$ & $\begin{array}{c}\text { Trayectoria } \\
1993-1998\end{array}$ & Patrón de reestructuración \\
\hline $\mathrm{I}$ & $\mathrm{I}$ & \\
\hline II & II & \\
\hline III & III & \\
\hline IV & IV & \\
\hline III & I & $\begin{array}{l}\text { Reestructuración drástica hacia la } \\
\text { expansión }\end{array}$ \\
\hline $\mathrm{I}$ & III & $\begin{array}{l}\text { Reestructuración drástica hacia el } \\
\text { retroceso }\end{array}$ \\
\hline II & IV & $\begin{array}{l}\text { De la transformación radical a la } \\
\text { progresiva }\end{array}$ \\
\hline IV & II & $\begin{array}{l}\text { De la transformación progresiva a } \\
\text { la radical }\end{array}$ \\
\hline II & $\mathrm{I}$ & Éxito de la transformación radical \\
\hline II & III & $\begin{array}{l}\text { Fracaso de la transformación } \\
\text { radical }\end{array}$ \\
\hline IV & I & $\begin{array}{l}\text { Éxito de la transformación } \\
\text { progresiva }\end{array}$ \\
\hline IV & III & $\begin{array}{l}\text { Fracaso de la transformación } \\
\text { progresiva }\end{array}$ \\
\hline I & II & $\begin{array}{l}\text { De la expansión a la } \\
\text { transformación radical }\end{array}$ \\
\hline I & IV & $\begin{array}{l}\text { De la expansión a la } \\
\text { transformación radical }\end{array}$ \\
\hline III & II & $\begin{array}{l}\text { Del retroceso a la transformación } \\
\text { progresiva }\end{array}$ \\
\hline III & IV & $\begin{array}{l}\text { Del retroceso a la transformación } \\
\text { progresiva }\end{array}$ \\
\hline
\end{tabular}

Fuente: Elaboración propia con base en tabla 2. 
Tabla 4

Trayectorias y patrones de reestructuración de las ramas seleccionadas de la ICHAB

\begin{tabular}{|c|c|c|c|c|}
\hline Municipio & Rama & \begin{tabular}{|c|} 
Trayectoria \\
$1988-1993$ \\
\end{tabular} & \begin{tabular}{|c|} 
Trayectoria \\
$1993-1998$ \\
\end{tabular} & Patrón de reestructuración \\
\hline Comitán & 3121 & I & I & \\
\hline Tapachula & 3113 & III & III & \\
\hline Comitán & 3112 & III & $\mathrm{I}$ & Reestructuración drástica hacia la expansión \\
\hline San Cristóbal de Las Casas & 3112 & III & $\mathrm{I}$ & Reestructuración drástica hacia la expansión \\
\hline Tuxtla Gutiérrez & 3130 & III & $\mathrm{I}$ & Reestructuración drástica hacia la expansión \\
\hline Tapachula & 3114 & $\mathrm{I}$ & III & Reestructuración drástica hacia el retroceso \\
\hline San Cristóbal de Las Casas & 3130 & I & III & Reestructuración drástica hacia el retroceso \\
\hline Comitán & 3115 & II & IV & De la transformación radical a la progresiva \\
\hline Tuxtla Gutiérrez & 3115 & II & IV & De la transformación radical a la progresiva \\
\hline Tapachula & 3130 & II & IV & De la transformación radical a la progresiva \\
\hline Tapachula & 3112 & IV & II & De la transformación progresiva a la radical \\
\hline Comitán & 3114 & IV & II & De la transformación progresiva a la radical \\
\hline Tapachula & 3121 & IV & II & De la transformación progresiva a la radical \\
\hline Comitán & 3130 & IV & II & De la transformación progresiva a la radical \\
\hline Tapachula & 3115 & II & $\mathrm{I}$ & Éxito de la transformación radical \\
\hline Tuxtla Gutiérrez & 3119 & II & III & Fracaso de la transformación radical \\
\hline Tuxtla Gutiérrez & 3121 & IV & $\mathrm{I}$ & Éxito de la transformación progresiva \\
\hline Tuxtla Gutiérrez & 3112 & IV & III & Fracaso de la transformación progresiva \\
\hline San Cristóbal de Las Casas & 3114 & IV & III & Fracaso de la transformación progresiva \\
\hline San Cristóbal de Las Casas & 3115 & $\mathrm{I}$ & IV & De la expansión a la transformación progresiva \\
\hline San Cristóbal de Las Casas & 3121 & $\mathrm{I}$ & IV & De la expansión a la transformación progresiva \\
\hline Tuxtla Gutiérrez & 3114 & III & II & Del retroceso a la transformación radical \\
\hline Comitán & 3119 & & II & \\
\hline San Cristóbal de Las Casas & 3119 & & III & \\
\hline
\end{tabular}

Fuente: Elaboración propia con base en los resultados del sistema Indica 1.0 


\section{Notas}

${ }^{1}$ Agradezco a Fredy Jiménez López y Victoria Jiménez Cruz la colaboración en el procesamiento de la información estadística.

${ }^{2}$ Estos censos se publicaron en 1989, 1994 y 1999, respectivamente.

${ }^{3}$ El café soluble se clasifica bajo la clase 312110 Elaboración de café soluble. Incluye extractos y concentrados de café.

${ }^{4}$ Los cálculos se llevaron a cabo mediante el sistema Indica 1. 0 (Morales, 2003).

\section{Bibliografía}

Camagni, Roberto y Roberta Capello, 1997, "Metodologie per la definizione del piano di riconversione regionale e sociale della Liguria: strategie e assi di intervento", en Bramanti, A. y M. Maggioni (eds.), La dinamica deisistemi produttivi territoriali: teorie, tecniche, politiche, Milano, Franco Angeli, pp. 399-423.

—, 1999, "Innovation and Performance of SMEs in Italy: The Relevance of Spatial Aspects", en Fischer, M. M. et al. (eds.), Innovation, Networks and Localities, Berlin, Springer-Verlag, pp. 181-214.

Camagni, Roberto y R. Cappellin, 1985, La productivité sectorielle et la politique régionale, Bruselas, Comisión de las comunidades Europeas.

Garrido, Rubén, 2002, Cambio estructural y desarrollo regional en España, Madrid, Pirámide.

Instituto Nacional de Estadística Geografía e Informática (INEGI), 1991, Censos económicos 1986 y 1989. Sistema automatizado de información censal, SAIC, Aguascalientes, INEGI.

—, 1993, Censos económicos 1989. Sistema automatizado de información censal, SAIC 2.0, Aguascalientes, INEGI.

—, 1994, Sistema de cuentas nacionales de México. Producto interno bruto por entidad federativa 1985 y 1988 , México, INEGI.

—, 1996, Censos económicos 1994. Sistema automatizado de información censal, SAIC 3.1, Aguascalientes, INEGI.
—, 2001, Censos económicos 1999. Sistema automatizado de información censal, SAIC 4, Aguascalientes, INEGI.

Llorens, Juan Luis et al., 2002, Estudios de caso de desarrollo económico local en América Latina, Washington, D.C., Banco Interamericano de Desarrollo, Serie de informes de buenas prácticas del Departamento de Desarrollo Sostenible.

Meyer-Stamer, Jörg, 1998, "Clustering, Systemic Competitiveness ad Commodity Chains: Shaping Competitive Advantages at the Local Level in Santa Catarina/Brazil", Revised version of the Paper prepared for the International Workshop "Global Production and Local Jobs: New Perspectives on Enterprise Networks, Employment and Local Development Policy", International Institute for Labour Studies, Geneva, 9-10 March, http://www.mesopartner.org/

—, 2000, "Estrategias de Desarrollo Local y Regional: Clusters, Política de Localización y Competitividad Sistémica", El Mercado de Valores, septiembre, Nacional Financiera, pp. 18-31.

Morales, Federico, 2003, "Trayectoria y reestructuración económica. Una tipología de las economías municipales de Chiapas", Dinámica estructural en economías regionales subdesarrolladas: un marco de interpretación de la relación globallocal, Reporte final de investigación, San Cristóbal de Las Casas, Proimmse-IIA-UnAM, tomo 1, pp. 6-157 y tomo 2, Anexo.

—, 2004, "Trayectoria y reestructuración económica en los municipios de Chiapas", Coloquio internacional Chiapas: 10 años después, agosto, San Cristóbal de Las Casas, Chiapas, http://proimmse.unam.mx/doctos_baja.html.

Morgan, Kevin y Claire Nauwelaers, 2003, Regional Innovation Strategies. The Challenge for Less-Favoured Regions, London, Routledge.

Nooteboom, Bart, 2000, Learning and Innovation in Organizations and Economies, Oxford, Oxford University Press.

Ratti, Remigio et al. (eds.), 1997, The Dynamics of Innovative Regions. The GREMI Approach, Aldershot, Ashgate.

Vázquez, Antonio, 1999, "Desarrollo, redes e innovación. Lecciones sobre desarrollo endógeno", Madrid, Pirámide. 